\title{
Gaze Data Visualizations for Educational VR Applications
}

\author{
Yitoshee Rahman \\ University of Louisiana at Laffayette \\ yitoshee.rahman1@louisiana.edu
}

\author{
Sarker Monojit Asish \\ University of Louisiana at Laffayette \\ C00304931@louisiana.edu
}

\author{
Adil Khokhar \\ University of Louisiana at Laffayette \\ C00016932@louisiana.edu
}

\author{
Arun K. Kulshreshth \\ University of Louisiana at Laffayette \\ arunkul@louisiana.edu
}

\author{
Christoph W. Borst \\ University of Louisiana at Laffayette \\ cwborst@gmail.com
}

\begin{abstract}
VR displays (HMDs) with embedded eye trackers could enable better teacher-guided VR applications since eye tracking could provide insights into student's activities and behavior patterns. We present several techniques to visualize eye-gaze data of the students to help a teacher gauge student attention level. A teacher could then better guide students to focus on the object of interest in the VR environment if their attention drifts and they get distracted or confused.
\end{abstract}

\section{CCS CONCEPTS}

- Human-centered computing $\rightarrow$ Visualization techniques.

\section{KEYWORDS}

Virtual Reality, Eye tracking, Educational VR, Gaze data visualization, User Experience.

ACM Reference Format:

Yitoshee Rahman, Sarker Monojit Asish, Adil Khokhar, Arun K. Kulshreshth, and Christoph W. Borst. 2019. Gaze Data Visualizations for Educational VR Applications. In Symposium on Spatial User Interaction (SUI '19), October 19-20, 2019, New Orleans, LA, USA. ACM, New York, NY, USA, 2 pages. https://doi.org/10.1145/3357251.3358752

\section{INTRODUCTION}

Virtual reality has long been suggested as a way to enhance education [5]. VR displays (HMDs) could be used for remote virtual reality based educational applications (e.g., a virtual lab, a virtual field trip) where a teacher could remotely guide a group of students. VR can produce experiences that are vividly remembered, along with numerous other effects that seem to hinge on immersive or embodied experiences [1]. However, there are distractions in VR which students may shift focus away from the main educational information [2]. We can mitigate such problems with use of students eye-gaze data obtained from the HMD's embedded eye-tracker (e.g., HTC Vive Pro Eye). Eye-gaze data could help a teacher identify confused/distracted students and then a teacher could better guide those students towards the object of interest in the VR environment. This work explores different gaze-data visualization techniques for

Permission to make digital or hard copies of part or all of this work for personal or classroom use is granted without fee provided that copies are not made or distributed for profit or commercial advantage and that copies bear this notice and the full citation on the first page. Copyrights for third-party components of this work must be honored.

For all other uses, contact the owner/author(s).

SUI '19, October 19-20, 2019, New Orleans, LA, USA

(C) 2019 Copyright held by the owner/author(s).

ACM ISBN 978-1-4503-6975-6/19/10.

https://doi.org/10.1145/3357251.3358752 the teacher and these visualizations would be the basis of identifying students who need additional help to direct or maintain their attention. These techniques would let a teacher visualize real-time eye tracking data which will help understand the attention level of students and improve the effectiveness of such VR system to supervise and assist students.

Eye tracking devices record gaze points of a participant as raw data which can be then aggregated into fixations and saccades, the two major categories of eye movement. A fixation is an aggregation of gaze points based on a specified area and time span. A saccade is described as a rapid eye movements from one fixation to another. Researchers have used line charts, bar charts, coordinate plots and scatter plots to visualize fixation data [3, 4]. However, these conventional visualization are not ideal for the VR environment. We explored techniques that may be more suitable for gaze-data visualization in a VR environment.

\section{GAZE DATA VISUALIZATIONS}

We designed and implemented several gaze data visualization techniques for the teacher, as follows (see Figure 1):

Gaze Circle (GC): Each colored circle represents the current fixation point of a student. Smoothing over time is applied to reduce jitter in the gaze data.

Gaze Circle with Student Position (GCP): This technique represents a student's position with a head model and a gaze circle is used to show where the student is looking.

Gaze Arrow with Student Position (GAP): A head model represents a student's position and an arrow is rendered to show the direction where a student is currently looking.

Gaze Trail (GT): A gaze-trail (saccade) is visualized over a time-span and each student is represented with a unique color. This technique not only shows where a student is looking currently, but also shows the history in a given timespan.

Gaze Trail with Arrows (GTA): Similar to gaze trail but an arrow indicating the direction of fixation point movement is also added. This allows a teacher to find out the sequence in which a set of objects is visualized.

Glowing Gaze Trail (GGT): Similar to gaze trail but it renders the trail using a particle effect. The particles show the history which interpolates from yellow to green over time. This creates an effect similar to arrows (as in GTA described above). 


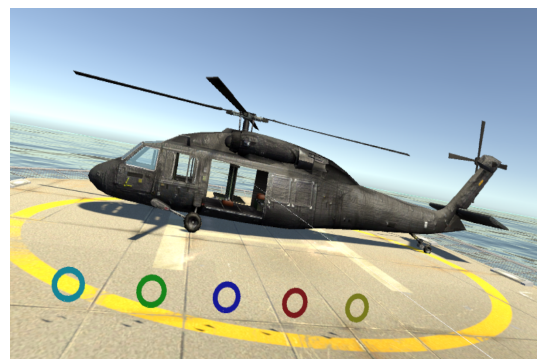

(a) GC

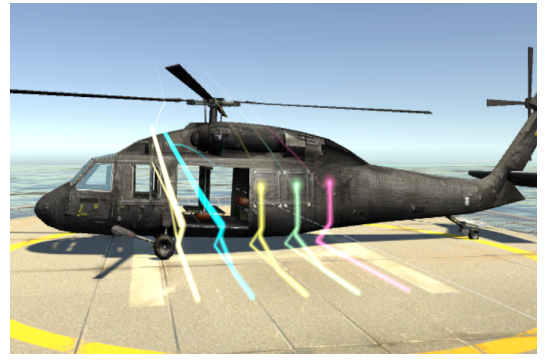

(d) GT

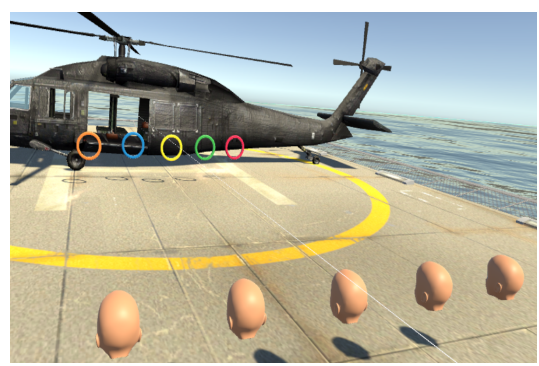

(b) GCP

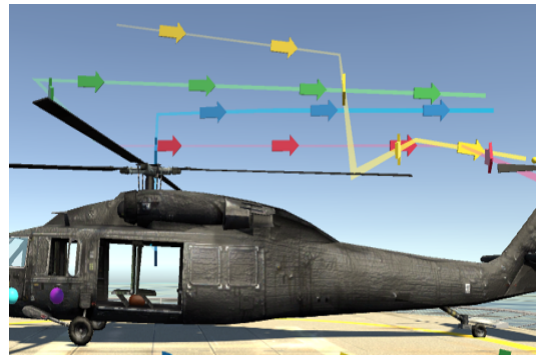

(e) GTA

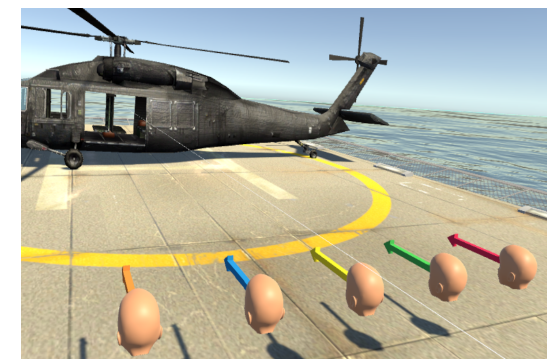

(c) GAP

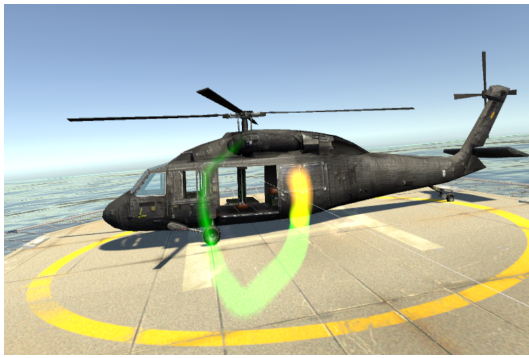

(f) GGT

Figure 1: Gaze data visualization techniques for a teacher.

\section{PRELIMINARY STUDY RESULTS}

We did a preliminary study with 9 participants. They rated each technique on a scale from 1 to 7 for accuracy (detecting where students are looking at), preference, frustration, and mental/cognitive demand. The results are summarized in Figure 2. The Gaze trail technique was not preferred by our participants since it had higher mental demand to accurately identify where the user is looking. Frustration levels were low with all the techniques. Glowing gaze trail was the most preferred technique. However, statistical significance was not assessed and we consider these results to just give a first impression.

\section{CONCLUSION AND FUTURE PLANS}

The goal of this work is to find out intuitive ways to visualize student's eye tracking data to help a teacher identify confused/distracted

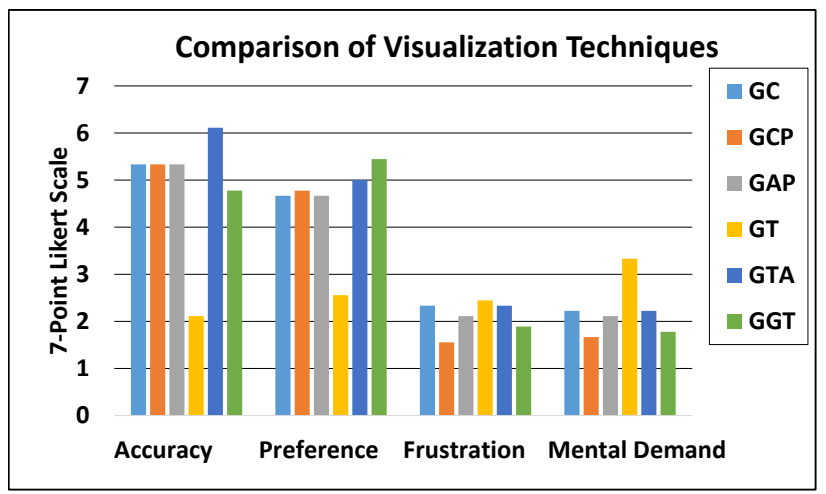

Figure 2: Summary of Results students in an immersive educational VR environment. Our preliminary results seem promising and would help us further improve these techniques. Possibly, some of these visualization techniques could be combined to design hybrids of these techniques. For example, gaze arrow with student position (GAP) could be combined with gaze circles to show the fixation area as well. For the future, we plan to design techniques to gauge attention level of students and conduct a usability experiment with detailed analysis of these techniques. Furthermore, we also plan to explore 3D heat maps for gaze data visualization.

\section{ACKNOWLEDGMENTS}

This material is based upon work supported by the National Science Foundation under Grant No. 1815976 and by the Louisiana Board of Regents under contract No. LEQSF(2019-20)-ENH-DE-22.

\section{REFERENCES}

[1] Jim Blascovich and Jeremy Bailenson. 2011. Infinite reality: Avatars, eternal life, new worlds, and the dawn of the virtual revolution. William Morrow \& Co.

[2] Christoph W Borst, Nicholas G Lipari, and Jason W Woodworth. 2018. Teacherguided educational VR: Assessment of live and prerecorded teachers guiding virtual field trips. In 2018 IEEE Conference on Virtual Reality and 3D User Interfaces (VR). IEEE, 467-474.

[3] Gregorio Convertino, Jian Chen, Beth Yost, Y-S Ryu, and Chris North. 2003. Exploring context switching and cognition in dual-view coordinated visualizations. In Proceedings International Conference on Coordinated and Multiple Views in Exploratory Visualization-CMV 2003-. IEEE, 55-62.

[4] Tim J Smith and Parag K Mital. 2013. Attentional synchrony and the influence of viewing task on gaze behavior in static and dynamic scenes. Fournal of vision 13 , 8 (2013), 16-16.

[5] Christine Youngblut. 1998. Educational uses of virtual reality technology. Technical Report. Institute for defense analysis, Alexendria VA. 